\title{
Transformation of a Sketch Map Laying over a Metric Map on the Web
}

\author{
Ryoto Kitajima and Ushio Inoue
}

\begin{abstract}
A sketch map is a type of non-metric map that is widely used for navigation and guidance. It is easy to understand, but the positions of geographical objects are not always accurate. This paper proposes a method to overlay a sketch map on a metric map so that each important object on the sketch map is located at the accurate position on the metric map. The method deforms the sketch map globally and locally with affine transformations, as well as adjusting the scale and heading of the metric map. By this way, users can get the best of both sketch maps that are easy to understand and metric maps that are accurate on locations. Our evaluation using 100 sketch maps on web pages demonstrates the effectiveness of the proposed method.
\end{abstract}

Index Terms-Geographical information systems, map overlay, map transformation, metric maps, sketch maps.

\section{INTRODUCTION}

In the present days, people commonly use digital maps on the Web to locate objects and find routes. Digital maps can be classified into metric maps and non-metric maps. A metric map is based on exact surveys and measurements. It draws every geographical object that satisfies predefined criteria at the accurate position based on a standard coordinate system. On the other hand, a non-metric map is based on human spatial cognition. It draws only objects of interest at an appropriate position. A sketch map is a type of non-metric maps, which are widely used for mainly navigation and guidance purposes. Before computers became popular, people used to write sketch maps on paper by hand, but nowadays, many people use drawing software tools on computer to draw sketch maps as digital images.

With the wide spread of the Internet technologies, metric maps and sketch maps are very common contents on the Web. Google and Yahoo provide online interactive mapping services by using metric maps. They are convenient for finding geographical locations of objects such as shops and restaurants, or getting directions to visit the place. However, a metric map is likely to show so many objects that users may feel difficulty in using the map to check their destinations or routes. As opposed to metric maps, sketch maps are simple and easy to understand. A sketch map shows only a small number of objects important for users, and omits or simplifies unimportant objects. Although sketch maps may not show objects at accurate positions, they are helpful to inform users

Manuscript received September 3, 2013; revised December 31, 2013.

Ryoto Kitajima was with Graduate School of Engineering, Tokyo Denki University, Tokyo, Japan. He is now with CyberAgent Inc., Tokyo, Japan (e-mail: kitajima.ryoto@gmail.com).

Ushio Inoue is with Tokyo Denki University, Tokyo, Japan (e-mail: inoue@c.dendai.ac.jp). about the locations and navigations of various places. For these reasons, many sketch maps are used in access pages on web sites.

Our goal of the research is to enhance utility values of sketch maps by matching them with metric maps. We propose a method to overlay a sketch map on a metric map so that geographical objects in the sketch map fit with the same objects in the metric map. Our method consists of five steps. In the first step, a sketch map is overlaid on a metric map, and then the size and scale of maps are adjusted. In the next step, important objects in the sketch map are roughly fitted to those in the metric map by changing the scale of the sketch map. The change of the scale is not uniform but depends on the portion of the sketch map. In the next step, objects in the sketch map unnecessary to be shown are made invisible. For example, roads written on the sketch map are unnecessary objects. In the next step, the sketch map is divided into a regular grid structure and each cell of the grid is individually transformed. In the final step, the transparency is controlled for the legibility. Thus, users can know the exact locations of objects that are shown in the sketch map, and check the absolute distances between objects.

The remainder of this paper is organized as follows; Section II reviews related work. Section III describes a web-based map system that we use in this research and common features of sketch maps. Section IV gives the design concepts and algorithms of our method. Section $\mathrm{V}$ shows the evaluation results by using a set of sketch maps collected from the Web. Section VI concludes this paper.

\section{RELATED WORK}

Many researchers have studied sketch maps from a variety of viewpoints. Their work can be mainly categorized into three groups.

The first group is studying the nature and effectiveness of sketch maps. Casakin et al. [1] studied spatial aspects of the physical environment such as branching points and connecting roads in the schematization of way-finding maps. Skubic et al. [2] proposed a methodology for extracting a qualitative model of a sketched route map, based on human navigation strategies using spatial relationships. Look and Shrobe [3] studied common elements found in people's mental maps of Boston city. Their analysis of hand-annotated maps suggested that prominent places and transit points play an important role. Meilinger et al. [4] empirically investigated different maps for way-finding tasks in a multi-level building. They concluded that providing unambiguous turning information rather than survey knowledge is most crucial for way-finding. Wang and Li [5] investigated three aspects of sketch maps for navigation, 
which are orientation, street topology and sequential order. They demonstrated sketch maps as a better direction-giving method than turn-by-turn instructions.

The second group is developing methods to generate sketch maps. Barkowsky et al. [6] presented a method for abstracting simplified cartographic representations, such as public transportation network maps from more accurate spatial data. Avelar et al. [7] proposed a method for preserving topological relations among linear features while generating sketch maps. Grabler et al. [8] and Kopf et al. [9] presented automated systems for designing two types of maps: tourist maps that selects and highlights the information important to tourists, and destination maps that are designed for navigation aids. Corcoran et al. [10] proposed a paradigm which exploits the geometric coherence in user requirements to reduce the computational cost associated with applying map transformation operators.

The third group is enhancing the value of sketch maps by integrating or comparing with metric maps. Schwering and Wang [11], [12] used sketch maps as a visual user interface for inserting spatial information into geographic information systems. They studied methods to establish a mapping between the sketched environment and the matching environment shown on a metric map. Kitayama et al. [13], [14] proposed a deformation analyzing method of sketch maps. Their method is based on geographical qualitative accuracy using the map information and surrounding text. Our research belongs to this group, but is different from the foregoing researches. We transform sketch maps directly in order to improve the geographical quantitative accuracy.

\section{METRIC MAPS AND SKETCH MAPS}

This section describes a web-based metric map system employed by our method and studies common features found in sketch map images on the Web. Then, an image transformation technique used for the map matching process is described.

\section{A. Metric Maps}

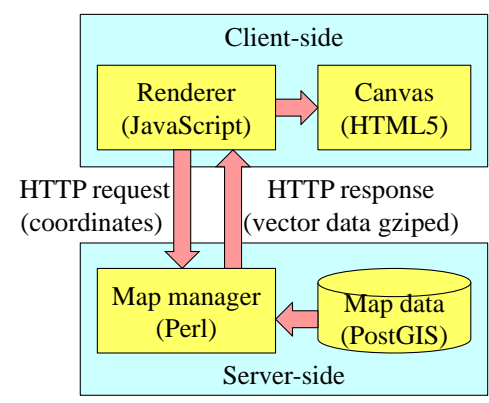

Fig. 1. Block diagram of CMap.

Interactive mapping systems currently available on the Web, such as Google Maps and Yahoo! Maps, produce metric maps on the server-side. In other words, the web server makes a picture of the map and sends it as an image file to a web browser, which displays it on the screen. This is a very versatile way because any kind of modern web browsers can be used. Our mapping system called CMap [15] employs a different way. Fig. 1 shows a block diagram of
CMap. The server sends vector data to the web browser, which draws a picture of the map on the client-side and displays it in the window. This is browser dependent because the browser must support JavaScript and HTML Canvas. However, the client-side drawing has several merits. First, it can change the scale and orientation of the map seamlessly not incrementally. Second, it can interact with users very smoothly because these change procedures are performed inside the client without communication with the server. Consequently, we decided to use CMap in this research.

\section{B. Sketch Maps}

The structure captured by sketch maps is not the structure of the physical world, but rather the conceptual structure of the information [16]. Sketch maps include important information such as landmarks and road signs, and eliminate the irrelevant such as unremarkable buildings and lanes. Moreover, sketch maps usually distort the spatial structure. They draw the area of interest with a large scale in detail and the peripheral area with a small scale in brief. They also simplify the shape of objects. For example, a curving road becomes straight, a corner becomes right-angled, and a block becomes rectangular. The orientation of the map is not always the north. Fig. 2 shows a sketch map of Tokyo Kanda campus of our university. We will use this map as an example throughout the paper.

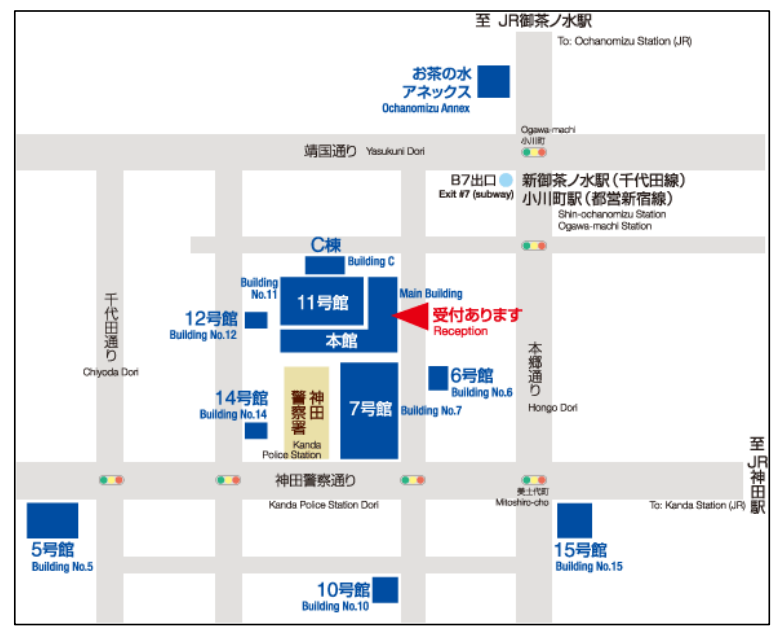

Fig. 2. Example of a sketch map.

\section{Affine Transformations}

In geometry, an affine transformation is a linear transformation including a translation. It is one of the most popular transformations in the image processing field. It has nice features for our sketch map transformation as follows:

- Points which lie on the same line (called collinear) continue to be collinear after the transformation.

- Ratios of distances between the collinear points continue to be same after the transformation.

If a point $(x, y)$ is mapped to another point $\left(x^{\prime}, y^{\prime}\right)$, the general affine transformation is commonly described by the following formula:

$$
\left(\begin{array}{l}
x^{\prime} \\
y^{\prime} \\
1
\end{array}\right)=\left(\begin{array}{lll}
a & b & c \\
d & e & f \\
0 & 0 & 1
\end{array}\right)\left(\begin{array}{l}
x \\
y \\
1
\end{array}\right),
$$


where $a, b, c, d, e$, and $f$ are transformation parameters.

If we know at least three coordinates of points inside a shape, we can identify these parameters. For example, suppose the triangle OAP is transformed to the triangle OAQ as shown in Fig. 3.

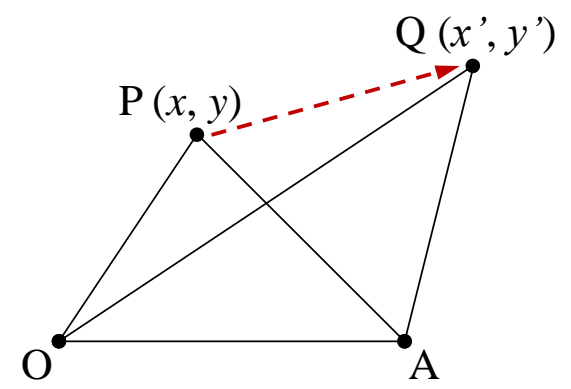

Fig. 3. Affine transformation of a triangle.

The coordinates of $\mathrm{O}, \mathrm{A}, \mathrm{P}$, and $\mathrm{Q}$ are defined as $(0,0),\left(x_{1}\right.$, $0),\left(x_{2}, y_{2}\right)$, and $\left(x_{2}, y_{2}{ }^{\prime}\right)$, respectively, without losing the generality. Then, any points $(x, y)$ inside the triangle OAP are mapped to another point $\left(x^{\prime}, y^{\prime}\right)$ in the triangle OAQ by using the following equation:

$$
\left(\begin{array}{l}
x^{\prime} \\
y^{\prime} \\
1
\end{array}\right)=\left(\begin{array}{lll}
1 & b & 0 \\
0 & e & 0 \\
0 & 0 & 1
\end{array}\right)\left(\begin{array}{l}
x \\
y \\
1
\end{array}\right),
$$

where $b=\left(x_{2}{ }^{\prime}-x_{2}\right) / y_{2}$ and $e=y_{2}^{\prime} / y_{2}$.

Our method divides a sketch map image into a grid structure, and then each cell of the grid is divided into triangles. The affine transformation is applied to each triangle when a user moves a vertex of the triangle by mouse.

\begin{tabular}{|c|c|}
\hline Sketch map transformation \\
\hline 5. Transparency control \\
\hline 4. Fine fitting \\
\hline 3. Removing unused obj. \\
\hline 2. Rough fitting \\
\hline 1. Placing maps \\
\hline $\begin{array}{c}\text { Adjusting position, } \\
\text { scale and orientation }\end{array}$ \\
\hline Metric map transformation \\
\hline
\end{tabular}

Fig. 4. Five steps of the proposed method.

\section{PROPOSED Method}

Our method is composed of five steps: placing maps, rough fitting, removing disused objects, fine fitting, and transparency control as shown in Fig. 4. This section explains each step in detail.

\section{A. Placing Maps}

The first step consists of three sub-steps and makes preparations for transformations of a sketch map in the following steps:

1) The system displays a metric map covering the area of interest on the browser window.
2) It reads a sketch map image file and displays the image over the metric map.

3) The user adjusts the position, scale and orientation of the metric map so that the sketch map is placed at a right position and direction on the metric map. A toolbar to control the transparency of the sketch map image is provided to help the adjustment process. Fig. 5 shows the result of this step.

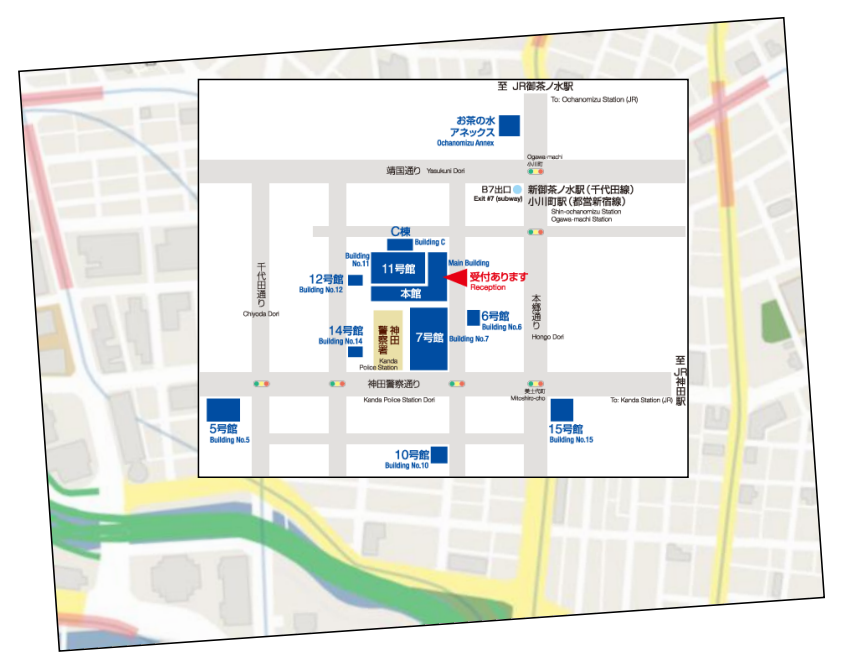

Fig. 5. Result of the placing maps.

\section{B. Rough Fitting}

The second step roughly fits important objects on the sketch map and those on the metric map. As mentioned before, sketch maps tend to describe the important area in the central part with a large scale. Therefore, we divide the sketch map into the central and peripheral parts and apply different scales to them. This step consists of following sub-steps:

1) The system displays a red rectangular frame at the center of the sketch map image, as shown in Fig. 6 a). The initial frame size is half as height and width as the sketch map image.

2) The user changes the scales of the central and peripheral parts of the sketch map by moving the red frame up-and-down or left-and-right. The transformation of each part of the map is performed by affine transformations described in Section III-c. Fig. 6 b). illustrates the result of this step, where the size of the center red frame is reduced.

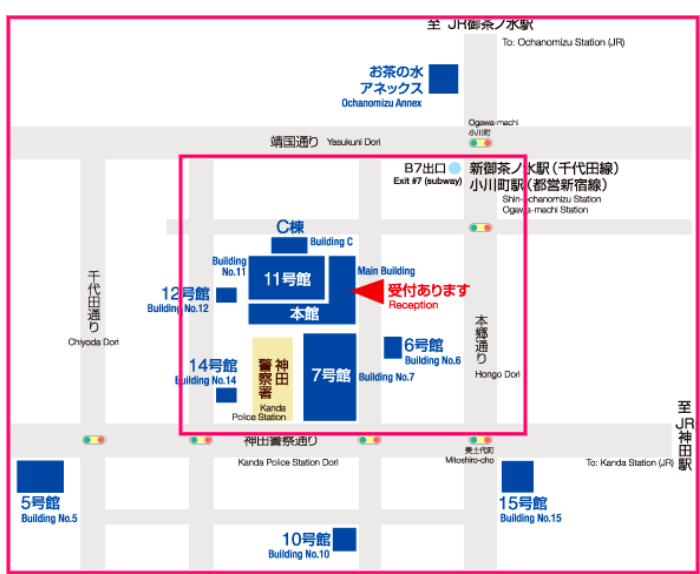

a) Before fitting. 


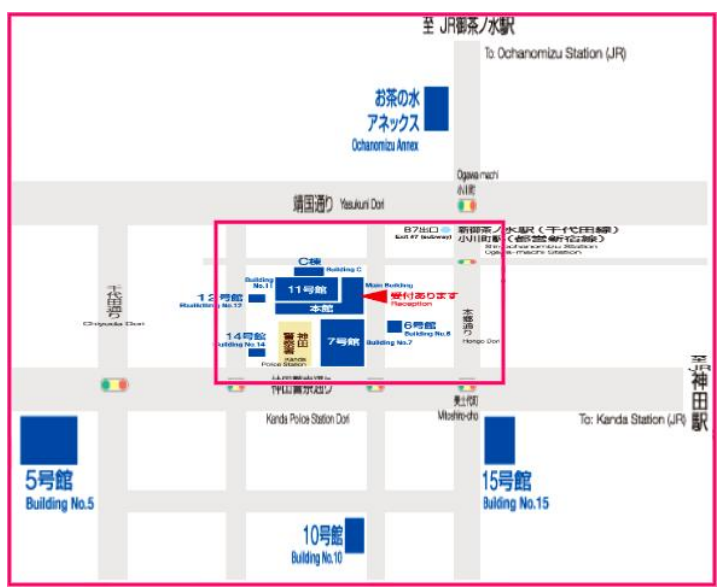

b) After fitting.

Fig. 6. Applying the rough fitting.

\section{Removing Disused Objects}

The third step removes unnecessary objects in the sketch map if necessary. Roads are unnecessary in most cases. The reason is that we can use accurate roads on the metric map instead of inaccurate roads on the sketch map. Another reason is that exact matching of the curves of roads in the next step can be very troublesome. This step consists of following sub-steps:

1) The user clicks a part of any road drawn on the sketch map.

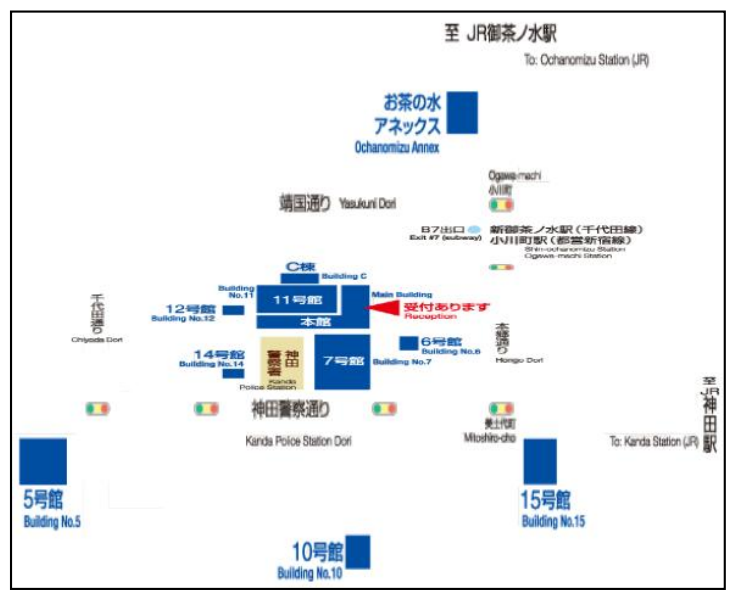

Fig. 7. Result of the removing unused objects.

2) The system gets the color information (RGB values) of the pixel.

3) The system makes every pixel in the image transparent, which has a similar color. The threshold value to determine the color similarity will be discussed in Section V. Fig. 7 shows the result of this step.

\section{Fine Fitting}

The fourth step divides the sketch map into a regular grid structure and transforms cells of the grid one by one. This step consists of following sub-steps:

1) The system displays red marks at a regular interval on the sketch map as shown in Fig. 8. Each mark is located at the corner or center of the grid cells, which is a mouse draggable point.

2) The user matches objects in the sketch map with those in the metric map by moving the marks to desired positions. The transformation of each cell is performed with a triangulation of the cell and affine transformations of triangles sharing the same vertex as shown in Fig. 9 a) and $9 \mathrm{~b})$.
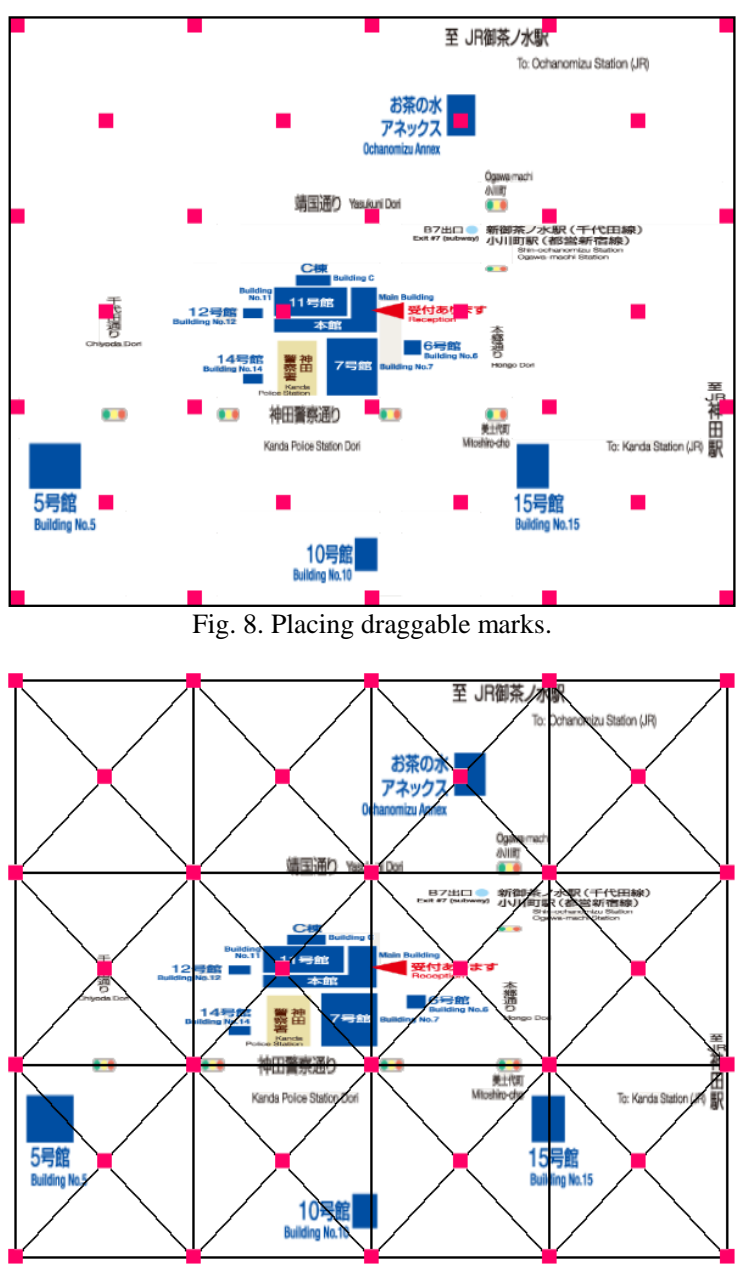

a) Triangulation

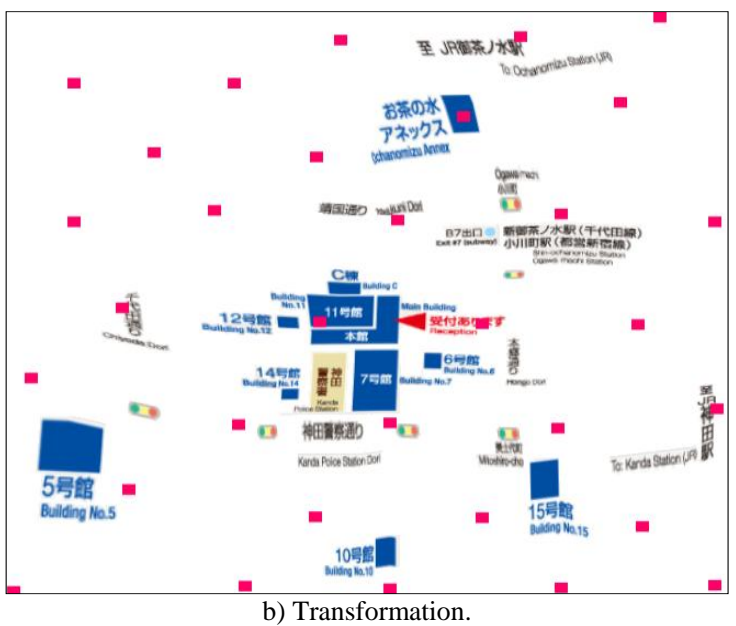

Fig. 9. Applying the fine fitting.

\section{E. Transparency Control}

The final step controls the transparency of the sketch map image by changing a value of the alpha channel of each pixel. The value is between 0 and 1 , where a value of 0 means that the pixel is completely transparent and a value of 1 means that the pixel is completely opaque. The user selects an appropriate value so that he/she can recognize objects in the both maps easily on the window. Fig. 10 illustrates the result of the step. 


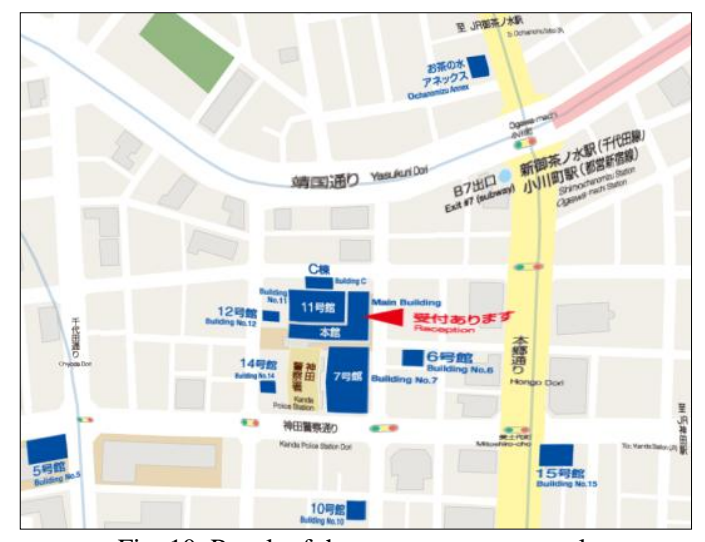

Fig. 10. Result of the transparency control.

\section{Evaluation}

We evaluated the proposed method from a viewpoint of location accuracy and required time. This section describes the experimental system, evaluation dataset, and evaluation results.

\section{A. Prototype System}

We constructed a prototype system for the evaluation. The system is an extension of CMap at the client-side as shown in Fig. 11. A sketch map image stored in the local storage is uploaded and overlaid on the canvas of CMap. It is transformed through the steps described in Section IV by user's manual operation. Some important parameters such as the grid size of the fine fitting are configurable for the execution.

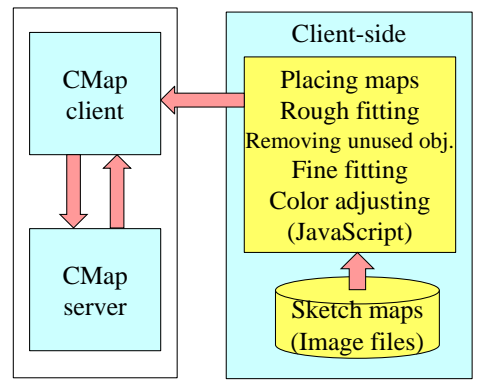

Fig. 11. Block diagram of the prototype system.

\section{B. Evaluation Dataset}
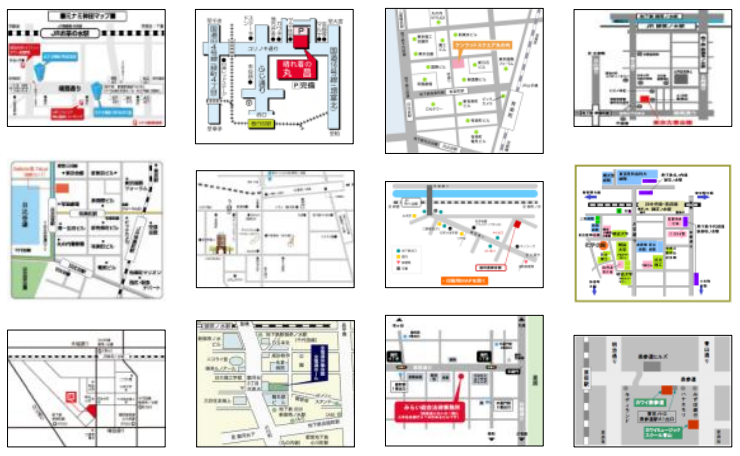

Fig. 12. Examples of sketch maps used.

We collected 100 sketch maps of several areas in Tokyo from the web. Google Image Search service (Google Images) was used to retrieve image files with keywords like "map Asakusa" (Asakusa is a very popular sightseeing area in Tokyo). We unintentionally selected sketch maps among the retrieved files excluding photo images, painted pictures, and topological maps (e.g. route map of subway). Sketch maps created by editing metric maps were also excluded, because the position of every object in such maps is so accurate and fits with the metric map naturally without transformations. Examples of sketch maps used are shown in Fig. 12.

\section{Evaluation Method}

In map making applications, triangulation is commonly used, which is the process of determining the location of a point by many triangles. Therefore, as a baseline method for our comparison, we transformed sketch maps solely by the fine fitting step described in Section IV.

We measured the accuracy and required time of the baseline and proposed methods by the following way. First, we divided every sketch maps into the $3 \times 3$ grid consists of 9 cells. Each cell has 4 triangles as shown in Fig. 9 a). Then we tried to fit objects in the sketch map with metric map by moving every vertex of triangles. If the location differences of important objects cannot be within a threshold, we divided the sketch map into $4 \times 4$ grid and tried the fitting process again. These procedures were repeated with increasing the grid size to $5 \times 5$ and $6 \times 6$ until the location difference is within the threshold. We defined the threshold as 45 pixels, which is the maximum permissible difference for most of the people.

\section{Evaluation Results}

The evaluation results on the location accuracy of sketch maps transformed with the baseline and proposed methods are shown in Fig. 13. It can be observed that most of the maps required the grid size $5 \times 5$ and more with the baseline, whereas the grid-size $4 \times 4$ and less with the proposed method. This means that time and labor for the map transformation was significantly saved by the proposed method.

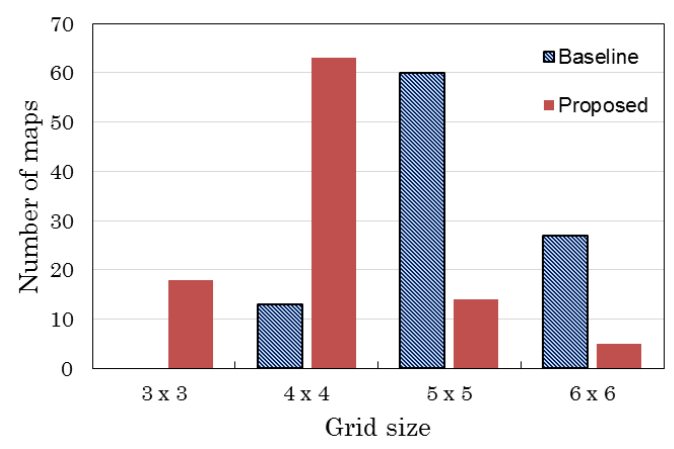

Fig. 13. Location accuracy of transformed sketch maps.

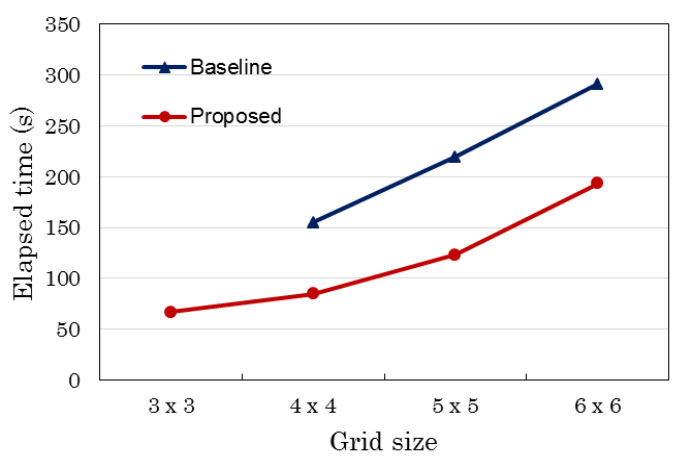

Fig. 14. Elapsed time for the map transformation. 
Fig. 14 shows the elapsed time required for the transformation which includes both human and machine processing time. As can be seen, the proposed method is shorter than the baseline in time at every grid size, though the elapsed time increases as the grid size increases.

Table I shows the average of the total time required in all of the steps for the 100 sketch maps. The proposed method enables the map transformation within less than half of the time required by the baseline method. This improvement is mainly caused by the rough fitting step, which reduces the grid size (the number of vertexes) as well as the distance of marks moved at the fine fitting step.

TABLE I: AVERAGE TIME FOR THE ENTIRE PROCESS

\begin{tabular}{c|c|c}
\hline \hline & Baseline method & Proposed method \\
\hline $\begin{array}{c}\text { Total time } \\
\text { (in second) }\end{array}$ & 230 & 92.5 \\
\hline \hline
\end{tabular}

\section{E. Considerations}

This subsection studies on two topics: the removing unused object step and the fine fitting step.

Removing unused object is optionally performed to make them invisible so that the user does not worry about matching errors about them. In most of sketch maps, every road is written in the same color, typically light gray or light blue. However, due to data compression techniques such as JPEG, each pixel value may be a little different with others. Therefore, our system needs to find pixels that have similar colors. If the selection is too strict, the road will not disappear On the other hand, if the selection is too loose, the other kind of objects such as buildings will disappear. We tested the 100 sketch maps by varying color difference allowance of pixel values. For example, let the pixel value which user selected is $(128,128,128)$ in RGB and the allowance is 3 . A pixel with $(131,130,126)$ in RGB should be invisible, but another pixel with $(132,130,126)$ in RGB should be visible because the value 132 exceeds the allowance. The evaluation result of removing road objects is shown in Fig. 15.

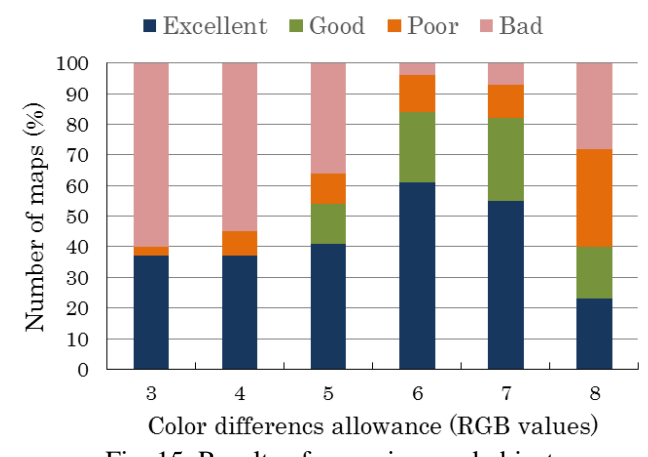

Fig. 15. Results of removing road objects.

In this figure, "Excellent" means that all roads were successfully removed; "Good" means that most of the roads were removed; "Poor" means that many roads remained visible or some of other objects were removed, and "Bad" means most of roads remained visible or many of other objects were removed. From these results, the optimal allowance value 6 was used in our evaluation.

The fine fitting process is a very troublesome task for users. If the user carefully performs the task with a lot of time, the location accuracy will be improved. However, some sketch maps are especially difficult to do the task. Sketch maps with the following characteristics have such difficulties:

- The buildings of interest are located in a large scale at the corner of the map. For this type of sketch maps, many marks need to be moved for long distance.

- There are only a few objects except nameless roads on the map. For this type of sketch maps, we can hardly identify the roads corresponding to which roads in the metric map.

The first problem may be solved at the rough fitting step by setting the initial red rectangular frame at any place with any size. The second problem may be solved at the fine fitting step by placing the initial marks for dragging at arbitrary positions such as at exact crossroads. These should be studied in the future.

\section{CONCLUSIONS}

This paper has proposed a method to overlay a sketch map on a metric map so that geographical objects in the sketch map fit with the same objects in the metric map. The proposed method was designed to achieve high location accuracy and to reduce required time. The evaluation using 100 sketch maps collected from the web showed that the proposed method transforms sketch maps with smaller grids than the baseline method. Thus, we can get higher location accuracy with shorter required time.

There are two other issues to be studied in the next stage. The first is to develop an automatic system that transforms sketch maps without handwork of users. By employing image analysis techniques, the system should automatically locate corresponding positions on the sketch map and metric map. The second is to improve the readability of sketch maps after the transformation. The current method transforms character strings on sketch maps, such as names of streets, stations, and buildings. It makes difficult for users to read the strings after the transformation. By employing a character recognition technique, the system should separate strings not to be deformed from drawings to be deformed.

\section{ACKNOWLEDGMENT}

The authors wish to thank Kentaro Yajima and Yohei Shinoda for their support for developing our prototype system.

\section{REFERENCES}

[1] H. Casakin, T. Barkowsky, A. Klippel, and C. Freksa, "Schematic maps as wayfinding aids," Spatial Cognition II, Lecture Notes in Computer Science, no. 1849, Springer, 2000, pp. 54-71.

[2] M. Skubic, S. Blisard, C. Bailey, J. A. Adams, and P. Matsakis, "Qualitative analysis of sketched route maps: Translating a sketch into linguistic descriptions," IEEE Trans. on Systems, Man, and Cybernetics, vol. 34, no. 2, pp. 1275-1282, Apr. 2004.

[3] G. Look and H. Shrobe, "Towards intelligent mapping applications: A study of elements found in cognitive maps," in Proc. 12th Int. Conf. on Intelligent User Interfaces (IUI), Honolulu, 2007, pp. 309-312.

[4] T. Meilinger, C. Holscher, S. J. Buchner, and M. Brosamle, "How much information do you need? Schematic maps in wayfinding and self-localization," Spatial Cognition V Reasoning, Action, Interaction, Lecture Notes in Computer Science, no. 4387, Springer, 2007, pp. 381-400.

[5] J. Wang and R. Li, "An empirical study on pertinent aspects of sketch maps for navigation," in Proc. IEEE 11th Int. Conf. on Cognitive Informatics \& Cognitive Computing, 2012, pp. 130-139. 
[6] T. Barkowsky, L. J. Latecki, and K.-F. Richter, "Schematizing maps: Simplification of geographic shape by discrete curve evolution," Spatial Cognition II, Lecture Notes in Computer Science, no. 1849, Springer, 2000, pp. 41-53.

[7] S. Avelar, M. Muller, and M. Mfiller, "Generating topologically correct schematic maps," in Proc. 9th Int. Symp. on Spatial Data Handling, 2000, pp. 4-28.

[8] F. Grabler, M. Agrawala, R. W. Sumner, and M. Pauly, "Automatic generation of tourist maps," in Proc. ACM SIGGRAPH '08, ACM Trans. on Graphics, vol. 27, no. 3, article 100, Aug. 2008.

[9] J. Kopf, M. Agrawala, D. Bargeron, D. Salesin, and M. Cohen, "Automatic generation of destination maps," in Proc. ACM SIGGRAPH ASIA '10, ACM Trans. on Graphics, vol. 29, no. 6, article 158, Dec. 2010.

[10] P. Corcoran, P. Mooney, and M. Bertolotto, "Utilizing geometric coherence in the computation of map transformations," J. of Computers \& Geosciences, no. 47, pp. 151-159, Oct. 2012.

[11] Schwering and J. Wang, "SketchMapia - A framework for qualitative mapping of sketch maps and metric maps," in Proc. Las Navas 20th Anniversary Meeting on Cognitive and Linguistic Aspects of Geographic Spaces, 2010, pp. 1-17.

[12] M. Chipofya, J. Wang, and A. Schwering, "Towards cognitively plausible spatial representations for sketch map alignment," Spatial Information Theory, Lecture Notes in Computer Science, no. 6899, Springer, 2011, pp. 20-39.

[13] D. Kitayama, R. Lee, and K. Sumiya, "Deformation analysis of modified maps based on geographical accuracy and spatial context," in Proc. 44th Hawaii Int. Conf. on System Sciences (HICSS), 2011, pp. 1-9.

[14] D. Kitayama and K. Sumiya, "A deformation analysis method for artificial maps based on geographical accuracy and its application," in
Proc. 2nd Joint WICOW/AIRWeb Workshop on Web Quality (WebQuality), Lyon, 2012, pp. 19-26.

[15] K. Yajima Y. Yamazaki, and U. Inoue, "Proposal and implementation of WebGIS by client-side map drawing," in Proc. 1st Forum on Data Engineering and Information Management (DEIM), Kakegawa, Japan, 2009, article B1-2 (in Japanese).

[16] Tversky, "What do sketches say about thinking," AAAI Technical Report, SS-02-08, AAAI, 2002, pp. 148-151.

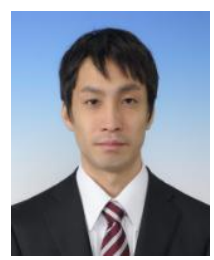

Ryoto Kitajima received his B.Eng. and M.Eng. degrees in information and communication engineering from Tokyo Denki University, Japan, in 2011 and 2013 respectively. He joined CyberAgent Inc. in 2013. His technical interests include web services and systems.

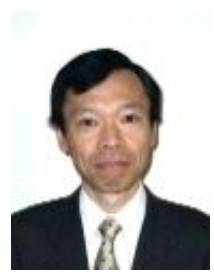

Ushio Inoue received his B.Eng. and D.Eng. degrees from Nagoya University, Japan in 1975 and 1992 respectively. In 1975, he joined NTT Laboratories, where he was engaged in research of information retrieval and database management systems. In 1995, he was a Senior Research Manager at NTT Data, where he developed distributed and multimedia information systems. Since 2004, he is a professor of Tokyo Denki University, Japan. Current his research interests include geographic information systems, information retrieval and recommendation, and education support systems. Prof. Inoue is a member of ACM, IEICE, IPSJ, and GISA. He is currently a Vice Chair of ACM SIGMOD Japan. 